\title{
STAR FORMATION AND MOLECULAR GAS IN NGC 404
}

\section{J. CEPA}

Instituto de Astrofísica de Canarias

E-38200 La Laguna, Tenerife, Spain

Departamento de Astrofísica

Universidad de La Laguna, E-38071 La Laguna, Tenerife, Spain

AND

B. VILA, N. NAKAI, K. KOHNO AND R. KAWABE

Nobeyama Radio Observatory

Minamimaki, Minamisaku, Nagano 384-13, Japan

\section{Introduction}

The last decade has brought about a completely different picture of elliptical galaxies. The once considered completely inert systems have revealed the presence of fair amount of dust, cool and cold gas, and undergoing star formation. The current statistics is that $80 \%$ of elliptical galaxies have detectable cool gas components, and as many as $60 \%$ have ionized gas (Knapp et al. 1989, Goudfrooij et al. 1994). Several single-dish CO surveys have been reported in the literature, allowing the deduction of several general properties of the molecular gas in early-type galaxies (Sage \& Wrobel 1989, Lees et al. 1991, Wiklind et al. 1995).

\section{This project}

As part of the survey on the star-forming activity in early-type galaxies that we have been carrying out, we present here the results of a preliminary analysis of the observations of the lenticular galaxy NGC 404 in B, $\mathrm{V}, \mathrm{I}, \mathrm{H} \alpha$ and $\mathrm{H} \beta$, optical spectroscopy and ${ }^{12} \mathrm{CO} \mathrm{J}=1 \longrightarrow 0$ Nobeyama Millimeter Array maps with a resolution of $3^{\prime \prime}$. NIR $(\mathrm{J}, \mathrm{H}, \mathrm{K})$ and $21 \mathrm{~cm}$ VLA observations are being processed and will provide additional clues to the star formation scenario sketched here. 


\section{Results}

\subsection{MOLECULAR GAS AND BROAD BAND DATA}

The $\mathrm{C}+\mathrm{D}$ combined data show a distribution peaking at a position offset by $3^{\prime \prime}$ to the SE of the optical nucleus. The distribution is clearly elongated in the NS direction and encompasses the $\mathrm{H} \alpha$ emitting regions. On the other hand, the $\mathrm{C}$ data alone shows a completely different morphology, peaking at the optical center (hosting as LINER as confirmed by the optical spectroscopy) and having an adjacent structure aligned with the dust lane clearly seen in the B, and especially in the B-I map. There is no clear rotation curve or systematic gradients in the velocity field or velocity dispersions, which suggests that the gas is likely a face-on disk. Using the standard conversion factor, we derive a total mass of $4.710^{6} \mathrm{M}_{\odot}(100 \%$ of the CO mass derived by Sage 1990, using the 12m NRAO antenna), and an average mass surface density of $26 \mathrm{M}_{\odot} \mathrm{pc}^{-2}$. This is clearly smaller than the gas amounts in spirals and also is an order of magnitude smaller than the neutral gas mass in this object (Hutchmeier \& Richter 1990).

\subsection{IONIZED GAS}

The $\mathrm{H} \alpha$ emission extends $\simeq 15^{\prime \prime}$ to the $\mathrm{S}$ of the optical nucleus. There is a clear lack of emission to the $\mathrm{N}$ of the nucleus that is probably caused by the strong extinction due to the dust lane lying there. From the $\mathrm{H} \alpha$ luminosity we can derive both the current massive star formation rates (for $\mathrm{M}>10 \mathrm{M}_{\odot}$, Kennicutt 1983) and their SFE. The values obtained were, SFR $\simeq 10^{-11} \mathrm{yr}^{-1}$ compatible with those obtained for M 51 by Kuno et al. (1995) taking into account both the total molecular and atomic gas masses. The correction of the SFR and SFE to all masses give, respectively, $610^{-3}$ $\mathrm{M}_{\odot} \mathrm{yr}^{-1}$ and $410^{-10} \mathrm{yr}^{-1}$, only a factor 3 smaller than the values derived from the correlation of FIR luminosities and SFRs (Thronson \& Telesco 1986). This discrepancy may arise both, from the dust extinction in the northern part of the nucleus and the somewhat loose FIR-SFR correlation.

\section{References}

Goudfrooij, P., Hansen, L., Jørgensen, H.E., Hørgaard-Nielsen, H.U. (1994), A $6 A S S$, 105,341

Kennicutt, R.C. (1983), $A p J, 272,54$

Knapp, G.R., Guhathakurta, P., Kim, D.-W., Jura, M. (1989), ApJS, 70, 329

Kuno, N., Nakai, N., Handa, T., Sofue, Y. (1995), PASJ, 47, 745

Lees, J.F., Knapp, G.R., Rupen, M.P., Phillips, T.G. (1991), ApJ, 379, 177

Sage, L.J. (1990), $A \& A, \mathbf{2 3 9}, 125$

Sage, L.J., Wrobel, J.M. (1989), $A p J, 344,204$

Thronson, M.A., Telesco, C.M. (1986), ApJ, 311, 98

Wiklind, T., Combes, F., Henkel, C. (1995), $A \& A$, 297, 643 